\title{
Avaliação de Inseticidas no Controle de Sitophilus oryzae (L.) (Coleoptera: Curculionidae), e Rhyzopertha dominica (Fab.) (Coleoptera: Bostrichidae) em Milho Armazenado
}

\author{
Paulo R.V.S. Pereira ${ }^{1}$, Rui S. Furiatti ${ }^{2}$, Flávio A. Lazzari ${ }^{1}$ e Airton R. Pinto $\mathrm{Jr}^{1}$ \\ ${ }^{1}$ Departamento de Zoologia, Universidade Federal do Paraná, \\ Caixa postal 19020, 81531-970, Curitiba, PR. \\ ${ }^{2}$ Departamento de Agronomia, Universidade Estadual de Ponta Grossa, Pça. Santos \\ Andrade, s/n, Caixa postal 992, 84100-970, Ponta Grossa, PR.
}

An. Soc. Entomol. Brasil 26(3): 411-416 (1997)

Evaluation of Insecticides in the Control of Sitophilus oryzae (L.) (Coleoptera: Curculionidae), and Rhyzopertha dominica (Fab.) (Coleoptera: Bostrichidae) in Shelled Corn

\begin{abstract}
The efficacy of insecticides was determined in laboratory against Sitophilus oryzae (L.) (Coleoptera: Curculionidae) and Rhyzopertha dominica (Fab.) (Coleoptera: Bostrichidae) exposed to treated shelled corn (Zea mays). Each treatment, which was composed by $1.2 \mathrm{~kg}$ of shelled corn, was treated with $5 \mathrm{ml}$ of mixture $/ \mathrm{kg}$ of corn using a propelling air atomizer. Fifteen days after treatment $200 \mathrm{~g}$ of the treated shelled corn, from each treatment, were infested with 40 adult insects of each species and this procedure was repeated every 30 days until 180 days after treatment. The number of dead insects was counted 15 days after each infestation, so the evaluations were made at 30, 60, 90, 120, 150 and 180 days after the insecticide application. After 180 days esfenvalerate + fenitrothion + piperonyl butoxide, esfenvalerate + fenitrothion and fenirothion + deltamethrin + pyperonil butoxide were effective against $S$. oryzae and $R$. dominica. Fenitrothion was effective against $S$. oryzae and deltamethrin + piperonyl butoxide against $R$. dominica. Esfenvalerate + pyperonil butoxide were ineffective against $S$. oryzae and the same was observed with fenitrothion against $R$. dominica. The use of mixtures of organophosphorus plus pyrethroids were more effective against $S$. oryzae and $R$. dominica when compared with these insecticides used alone.
\end{abstract}

KEY WORDS: Insecta, chemical control, stored product pests.

RESUMO - Determinou-se em laboratório a eficiência de inseticidas contra Sitophilus oryzae (L.) (Coleoptera: Curculionidae) e Rhyzopertha dominica (Fab.) (Coleoptera: Bostrichidae) em milho (Zea mays) a granel tratado. Cada tratamento, constituído por $1,2 \mathrm{~kg}$ de milho, foi tratado por meio de um pulverizador equipado com bico pneumático usando-se $5 \mathrm{ml}$ de calda/kg de milho. Quinze dias após os tratamentos $200 \mathrm{~g}$ de milho, de cada tratamento, foram infestadas com 40 insetos adultos de cada espécie, sendo este procedimento repetido a cada trinta dias até completar 180 dias. A contagem do número de insetos mortos foi realizada 15 dias após cada uma das infestações, desta forma foram feitas avaliações aos 30, 60, 90, 120, 150 e 180 dias após a 
aplicação dos inseticidas. Após 180 dias esfenvalerate + fenitrothion + butóxido de piperonila, esfenvalerate + fenitrothion e fenitrothion + deltametrina + butóxido de piperonila foram eficientes contra $S$. oryzae e $R$. dominica. Fenitrothion foi eficiente contra $S$. oryzae e deltametrina + butóxido de piperonila contra $R$. dominica. Esfenvalerate + butóxido de piperonila e esfenvalerate mostraram-se ineficientes contra $S$. oryzae, o mesmo sendo observado para fenitrothion contra $R$. dominica. O uso de misturas de organofosforados e piretróides foi mais eficiente contra $S$. oryzae e $R$. dominica quando comparado com o uso isolado destes inseticidas.

PALAVRAS-CHAVE: Insecta, controle químico, pragas de produtos armazenados.

Estima-se que as perdas quantitativas anuais causadas por pragas durante o período de armazenamento de grãos são da ordem de $10 \%$ da produção mundial. Esta estimativa refere-se, apenas, ao ataque dos insetos ao embrião e ao endosperma das sementes, não entrando em consideração o aquecimento da massa de grãos provocada pela atividade dos insetos, o consequente ataque de fungos e também, a diminuição do valor nutritivo dos grãos (Almeida 1989). No Brasil as perdas por ataque de pragas chegam a $10 \%$ da produção armazenada, anualmente (Lorini 1993). No armazenamento de milho (Zea mays) em espiga, utilizando estruturas rústicas, como os paióis construídos de madeira, as perdas causadas por insetos tem sido enormes (Santos 1993).

Dentre as pragas que atacam grãos de milho durante o armazenamento, destacamse Sitophilus zeamays (Mots.), S. oryzae (L.) (Coleoptera: Curculionidae) e Rhyzopertha dominica (Fab.) (Coleoptera: Bostrichidae), sendo consideradas pragas primárias por terem a capacidade de perfurar o tegumento de sementes e grãos.

Bareth \& Gupta (1989), testaram seis inseticidas no controle de $R$. dominica, concluindo que deltametrina a 2 ppm mostrou a melhor eficiência contra este inseto, seguido por fenitrothion a $20 \mathrm{ppm}$. Demonstraram também que nenhum dos inseticidas alterou a germinação das sementes. Giga \& Zvoutete
(1990), trabalhando com milho infestado com S. zeamais, observaram que fenitrothion e deltametrina foram eficientes até 120 e 90 dias após o tratamento, respectivamente. Guedes \& Silva (1992), obtiveram bom controle de $S$. zeamais com deltametrina $(0,43 \mathrm{ppm}) \mathrm{em}$ milho armazenado até 180 dias após o tratamento.

Este trabalho teve por objetivo avaliar a eficiência de inseticidas no controle de $S$. oryzae e $R$. dominica em milho armazenado a granel.

\section{Material e Métodos}

O experimento foi realizado no laboratório de Pragas e Patógenos de Grãos Armazenados da Universidade Federal do Paraná (UFPR), onde foram mantidas as colônias de $S$. oryzae e de $R$. dominica a serem utilizadas neste experimento. $\mathrm{O}$ delineamento estatístico utilizado foi em blocos casualizados, composto por quatro blocos de 11 parcelas cada, mantidos em sala climatizada $(25 \pm 2$ $\left.{ }^{\circ} \mathrm{C}\right)$.

Os tratamentos foram efetuados com um pulverizador propulsionado a ar, equipado com bico pneumático. Em todos os tratamentos utilizou-se $5 \mathrm{ml}$ de calda por quilo de milho $(5,01 / \mathrm{t})$, exceto a testemunha onde foi aplicado apenas água. Para facilitar a homogeneização, os tratamentos foram efetuados dentro de sacos plásticos de $0,4 \mathrm{x}$ 
$0,5 \mathrm{~m}$.

Nos tratamentos foram utilizados fenitrothion (Sumigran ${ }^{\circledR} 500 \mathrm{CE}, 15 \mathrm{ml} / \mathrm{t}$ ); esfenvalerate (Sumidan ${ }^{\circledR} 25 \mathrm{CE}, 20 \mathrm{ml} / \mathrm{t}$ ) a mistura pronta deltametrina + butóxido de piperonila (K-obiol $\left.{ }^{\circledR} 25 \mathrm{CE}, 15 \mathrm{ml} / \mathrm{t}\right)$, a mistura de fenitrothion + esfenvalerate $(15+15$ e $20+20 \mathrm{ml} / \mathrm{t}$ ), a mistura fenitrothion + deltametrina + butóxido de piperonila $(15+15$ $\mathrm{ml} / \mathrm{t}$ ), a mistura de esfenvalerate + butóxido de piperonila $(10+2,22$ e $20+4,44 \mathrm{ml} / \mathrm{t})$ e a mistura pronta de esfenvalerate + fenitrothion + butóxido de piperonila (Sumigran Plus, 15 e $20 \mathrm{ml} / \mathrm{t}$ ) (Tabela 1).

Para cada tratamento utilizaram-se $1,2 \mathrm{~kg}$ de milho, que após tratados foram acondicionados em sacos de ráfia e armazenados em sala climatizada. A cada 15 dias, $50 \mathrm{~g}$ deste milho, acondicionados em embalagens plásticas de 0,5 1 dotadas de tampa perfurada, foram infestadas com 10 insetos adultos não sexados, de cada espécie, provenientes de diversos pontos do Estado do Paraná e mantidos em sala climatizada (25 \pm $2{ }^{\circ} \mathrm{C}$ ). A avaliação do número de insetos mortos foi feita 15 dias após cada infestação. Desta forma realizaram-se avaliações aos 30, $60,90,120,150$ e 180 dias após o tratamento.

A eficiência no controle de $S$. oryzae e $R$. dominica, foi atribuída aos inseticidas que apresentaram mortalidade superior a $80 \%$ e diferença estatística significativa da testemunha.

Os dados obtidos foram submetidos a análise de variância e as diferenças entre as médias tratadas pelo teste de Tukey a 5\%. A eficiência relativa dos inseticidas foi calculada segundo Abbott (1925).

\section{Resultados e Discussão}

No controle de $S$. oryzae, esfenvalerate + butóxido de piperonila $(10,0+2,22$ e $20,0+$ $4,44 \mathrm{ml} / \mathrm{t}$ ) e esfenvalerate mostraram-se ineficientes desde o início do experimento. Deltametrina + butóxido de piperonila foi eficiente até os 150 dias após a aplicação. Esfenvalerate + fenitrothion + butóxido de piperonila $(15$ e $20 \mathrm{ml} / \mathrm{t})$, fenitrothion + esfenvalerate $(15+15$ e $20+20 \mathrm{ml} / \mathrm{t})$, fenitrothion + deltametrina + butóxido de piperonila e fenitrothion, mostraram-se eficientes até 180 dias após a aplicação (Tabela 1).

Os resultados obtidos no controle de $S$. oryzae com deltametrina + butóxido de piperonila, eficientes até 150 dias após o tratamento, são semelhantes aos encontrados por Guedes \& Silva (1992) usando deltametrina, no entanto, diferem dos resultados obtidos por Islam et al. (1989) e Giga \& Zvoutete (1990), onde este inseticida mostrou-se eficiente até os 90 dias. Os resultados obtidos com fenitrothion são semelhantes aos encontrados por Guedes \& Silva (1992).

No controle de $R$. dominica, com exceção de fenitrothion, que não foi eficiente em nenhuma das avaliações, todos os demais tratamentos mostraram-se eficientes até os 120 dias após a aplicação. Aos 150 dias perderam a eficiência os tratamentos esfenvalerate + butóxido de piperonila $(10,0$ $+2,22$ e $20,0+4,44 \mathrm{ml} / \mathrm{t}$ ) e esfenvalerate. Aos 180 dias após a aplicação mantiveram a eficiência esfenvalerate + fenitrothion + butóxido de piperonila (15 e $20 \mathrm{ml} / \mathrm{t}$ ), fenitrothion + esfenvalerate $(15+15$ e $20+$ $20 \mathrm{ml} / \mathrm{t}$ ), fenitrothion + deltametrina + butóxido de piperonila e deltametrina + butóxido de piperonila, cuja ineficiência aos 30 dias pode ser atribuída a problemas na metodologia, já que nas outras avaliações este produto mostrou-se eficiente (Tabela 2).

Os resultados obtidos com fenitrothion diferem daqueles obtidos por Bareth \& Gupta (1989), que testando inseticidas contra $R$. dominica em trigo, registraram a eficiência deste inseticida. Utilizando deltametrina estes mesmos pesquisadores obtiveram resultados semelhantes aos deste trabalho, onde este inseticida mostrou-se eficiente até os 180 dias após o tratamento. A ineficiência do esfenvalerate aos 150 dias mostra uma certa instabilidade do produto a partir deste período de armazenamento, apesar deste recuperar a eficiência aos 180 dias.

Em linhas gerais os tratamentos mais 


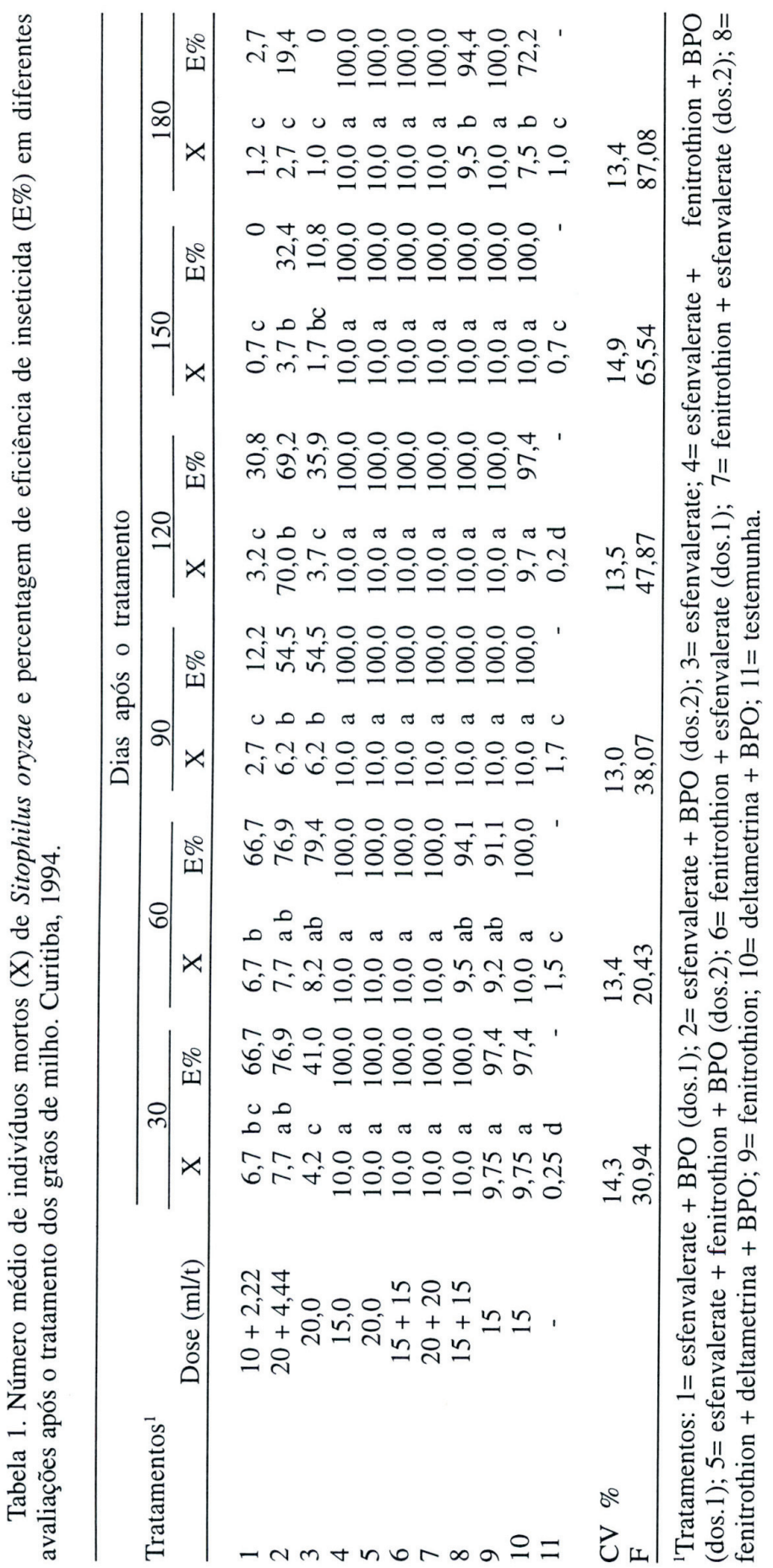




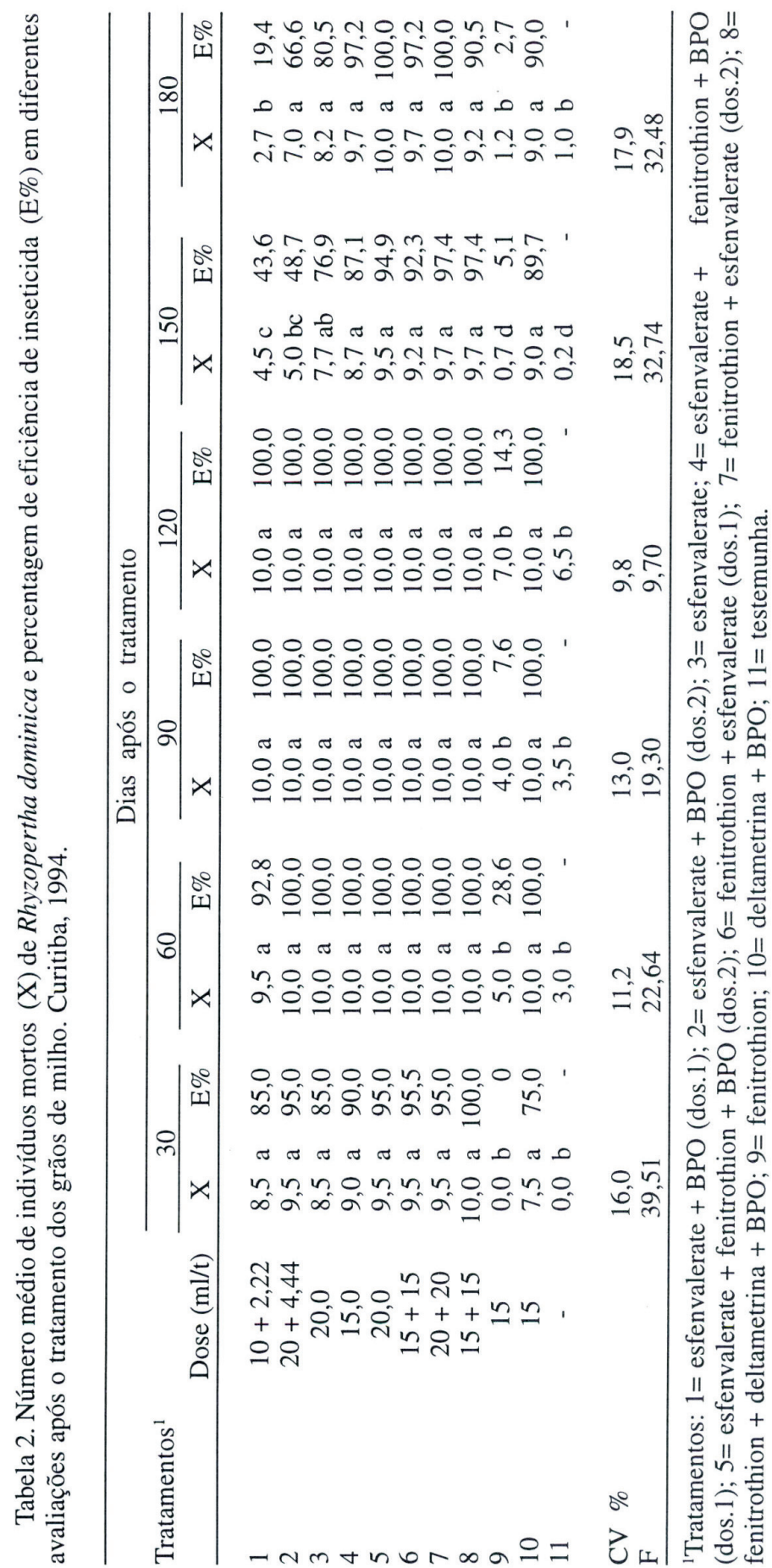


eficientes no controle de $S$. oryzae e $R$. dominica foram esfenvalerate + fenitrothion + butóxido de piperonila (15 e $20 \mathrm{ml} / \mathrm{t}$ ), fenitrothion + esfenvalerate $(15+15$ e $20+$ $20 \mathrm{ml} / \mathrm{t}$ ) e fenitrothion + deltametrina + butóxido de piperonila. Deltametrina + butóxido de piperonila mostrou-se eficiente contra $R$. dominica e fenitrothion contra $S$. oryzae.

Os tratamentos compostos por misturas de organofosforado (fenitrothion) e piretróides (esfenvalerate ou deltametrina) foram os que apresentaram melhores resultados, sendo eficientes durante os 180 dias de experimento, mantendo-se estáveis e com eficiência relativa próxima de $100 \%$. Estes mesmos produtos quando utilizados isoladamente mostraram eficiência para uma ou outra praga, mas não concomitantemente para as duas pragas estudadas.

Os resultados obtidos neste trabalho poderão ser úteis no controle de pragas de produtos armazenados. Entretanto, deve-se salientar que o uso de misturas de inseticidas pode favorecer o surgimento de populações resistentes aos grupos químicos envolvidos, dificultando um programa posterior de manejo desta resistência. A utilização de misturas só deve ser feita quando estritamente necessária, de maneira criteriosa e quando todos os fatores envolvidos no controle justifiquem seu uso.

\section{Literatura Citada}

Abbott, W.S. 1925. A method of computing the effectiveness of an insecticide. J. Econ. Entomol. 18: 265-267.

Almeida, A.A. 1989. Natureza dos danos causados por insetos em grãos armazenados. In: Anais Congr. Bras.
Entomol., 11, 1987, Campinas, Fund. Cargill v.4, p. 16-32.

Bareth, S.S. \& H.C.Gupta. 1989. Efficacy of six insectides for the protection of stored wheat seeds against Rhyzopertha dominica (Fab.). Seed Res. 17: 43-46.

Giga, D.P. \& P. Zvoutete. 1990. The evaluation of different insecticides for the protection of maize against some stored product pests. Int.Pest Control 32:10-13.

Guedes, R.N.C. \& F.A.P. da Silva. 1992. Avaliação do efeito residual do inseticida abamectin no controle de Sitophilus zeamais Motschulsky (Coleoptera: Curculionidae) em milho armazenado. Rev. Ceres 39: 435-442.

Islam, N., M.I.M. Bhuiyah, A. Begum \& M.A. Karim. 1989. Comparative efficacy of different materials against Sitophilus oryzae infesting maize seeds in storage. Bangladesh J. Zool. 17: 175178.

Lorini, I. 1993. Aplicação do manejo de integrado de pragas em grãos armazenados, p. 117 - 126. In Anais Simp. Prot. Grãos Armazenados, 1, Passo Fundo, RS, Embrapa - CNPT, 147 p.

Santos, J.P. 1993. Perdas causadas por insetos em grãos armazenados. p. 9 - 22. In Anais Simp. Prot. Grãos Armazenados, 1, Passo Fundo, RS, Embrapa - CNPT, $147 \mathrm{p}$.

Recebido em 10/07/96. Aceito em 17/08/96. 